\title{
Cambios alimentarios en Colombia; resultados de dos encuestas nacionales de nutrición, 2010-2015
}

\author{
Food changes in Colombia; results of two \\ national nutrition surveys, 2010-2015
}

\author{
Oscar-F Herrán ${ }^{1}$ Jhael-N Bermúdez²; María del Pilar Zea ${ }^{3}$
}

Forma de citar: Herrán OF, Bermúdez JN, Zea MP. Cambios alimentarios en Colombia; resultados de dos encuentas nacionales de nutrición, 2010-2015. Salud UIS. 2020; 52(1): 21-31. doi: http://dx.doi.org/10.18273/revsal.v52n1-2020004 @ (1)

\begin{abstract}
Resumen
Introducción: Colombia experimenta una transición alimentaria sumada a la nutricional. Objetivo: Establecer en Colombia para el período 2010-2015 la prevalencia y la frecuencia/día del consumo usual de 28 alimentos y dos prácticas de interés para la nutrición pública. Además, establecer las diferencias en la prevalencia y frecuencia/día entre años. Metodología: Estudio analítico en población entre 5 y 64 años, con datos de corte transversal recolectados en las dos últimas Encuestas Nacionales de Situación Nutricional en Colombia, ENSIN-2010 y 2015. Los datos de consumo fueron los obtenidos a través de un Cuestionario de Frecuencia de Consumo. A través de modelos de regresión lineal se estimaron las diferencias crudas y ajustadas para la prevalencia y la frecuencia/día del consumo usual de 28 alimentos y dos prácticas asociadas. Resultados: En el período 2010-2015, en los niños, la prevalencia de consumo de leche, carne, atún, vísceras, leguminosas, pan, tubérculos, azúcar, gaseosas y alimentos fritos disminuyó, al igual que la de verduras y frutas. En los adultos, la prevalencia de leche, carne, atún, vísceras, pan, tubérculos, azúcar, alimentos fritos y alimentos light disminuyó, al igual que la de verduras y frutas. En niños y adultos la prevalencia de consumo de alimentos de paquete aumentó, pero su frecuencia/día disminuyó. La prevalencia de uso del salero disminuyó en niños y adultos, pero su frecuencia/día aumentó. Discusión: El cambio alimentario establecido es similar en niños y adultos confirmando que la cultura alimentaria se hereda socialmente y se transmite de manera vertical entre padres e hijos. Conclusiones: Colombia experimenta una transición alimentaria. Se requiere política pública para orientar el consumo.
\end{abstract}

Palabras clave: Ingestión de alimentos; Niño; Adulto; Dieta; Encuestas nutricionales; Epidemiología; Prevalencia; Obesidad; Política pública; Colombia.

\footnotetext{
1. Universidad Industrial de Santander. Bucaramanga, Santander.

2. Instituto Colombiano de Bienestar Familiar. Bogotá, Colombia.

3. Universidad Javeriana de Cali. Valle del Cauca, Colombia.

Correspondencia: Oscar F. Herrán. Dirección: Escuela de Nutrición y Dietética, Facultad de Salud UIS, Carrera 32 No. 29-31. Teléfono: +5776323215. Correo electrónico: herran@uis.edu.co
} 


\begin{abstract}
Introduction: Colombia experiences a food transition coupled with nutritional transition. Objective: Establish in Colombia for the period 2010-2015 the prevalence and frequency/day of the usual consumption of 28 foods and two practices of interest for public nutrition. Also, establish differences in prevalence and frequency/day between years. Methodology: Analytical study in population between 5 and 64 years, with cross-sectional data collected in the last two National Surveys of Nutritional Situation in Colombia, ENSIN-2010 and 2015. The consumption data were obtained through a Food Frequency Questionnaire. Through linear regression models, raw and adjusted differences were estimated for the prevalence and frequency/day of usual consumption of 28 foods and two associated practices. Results: In the 2010-2015 period, in children, the prevalence of consumption of milk, meat, tuna, viscera, legumes, bread, tubers, sugar, soft drinks, and fried foods decreased, as did those of vegetables and fruits. In adults, the prevalence of milk, meat, tuna, offal, bread, tubers, sugar, fried foods, and light foods decreased, as did those of vegetables and fruits. In children and adults, the prevalence of packaged food consumption increased, but its frequency/day decreased. The prevalence of saltshaker use decreased in children and adults, but its frequency/day increased. Conclusions: The established dietary change is similar in children and adults confirming that the food culture is inherited socially and transmitted vertically between parents and children. Colombia experiences a food transition. Public policy is required to guide food consumption.
\end{abstract}

Keywords: Eating; Child; Adult; Diet; Nutrition surveys; Epidemiology; Prevalence; Obesity; Public policy; Colombia.

\section{Introducción}

El consumo de alimentos y los cambios en él han sido y siguen siendo de interés para la epidemiología nutricional ${ }^{1,2}$, ésta ha estudiado el consumo de nutrientes, alimentos, grupos de alimentos y patrones alimentarios para establecer la influencia de la dieta en el desarrollo de la enfermedad aguda y crónica. En Colombia en 2016, se estableció que además de la transición nutricional coexiste una transición alimentaria ${ }^{3}$. Los cambios alimentarios hasta ahora han sido asociados a lo que se denominó "transición nutricional" 4,5 . Sin embargo, las transiciones nutricional y alimentaria son fenómenos diferentes y complementarios. El exceso de peso y la obesidad son el efecto atribuible al consumo de alimentos sumado a otros factores estructurales denominados transiciones; demográfica, epidemiológica, económica, alimentaria ${ }^{3,4,6-8}$.

El cambio alimentario es insumo para monitorear y ajustar la política agraria y para evaluar la seguridad alimentaria ${ }^{9,10}$. El cambio no es homogéneo, está mediado por la estructura demográfica, económica, la política de precios en los alimentos y la capacidad de adaptarse de los países y grupos sociales al interior de los mismos ${ }^{10,11}$. El ajuste de las políticas de alimentación y nutrición debe, además, considerar la disminución en la emisión de gases de efecto invernadero ${ }^{12}$.

Colombia en las dos últimas Encuestas Nacionales de la Situación Nutricional (ENSIN, 2010 y 2015) aplicó la metodología de Cuestionario de Frecuencia de Consumo $(\mathrm{CFC})^{13}$, para estimar los cambios alimentarios, las prácticas asociadas y para establecer y ajustar la política de nutrición. El CFC es la metodología más costoefectiva y útil para aproximarse desde la epidemiología, tanto a la prevalencia como a la frecuencia/día del consumo usual. El CFC aplicado en Colombia cuenta con estudios de reproducibilidad y validez ${ }^{15,16}$ y la lista de alimentos se basó en los problemas de nutrición pública identificados en la ENSIN-2005 ${ }^{13,14}$.

Los objetivos del estudio fueron, a) establecer en el quinquenio 2010-2015 la prevalencia y la frecuencia/ día de consumo usual de algunos alimentos, grupos de alimentos y las prácticas asociadas al consumo alimentario en la población colombiana y b) establecer en el consumo las diferencias crudas y ajustadas por los principales determinantes, biológicos, económicos y sociales del mismo.

\section{Materiales y métodos}

Estudio analítico con base en datos de corte transversal recolectados en dos encuestas nacionales de nutrición, 2010 y 2015, que establecieron el consumo de alimentos y la realización de prácticas de interés en nutrición pública de la población colombiana.

\section{Población estudiada y muestra}

Colombia durante los últimos 10 años realizó dos ENSIN, 2010 y 2015. Los detalles de estas ENSIN ya fueron publicados ${ }^{14}$. En resumen, las ENSIN son diseñadas para representar al $99 \%$ de la población, a través muestreo 
polietápico estratificado. En 2010 la encuesta incluyó 50.670 hogares, distribuidos en 4.987 clúster de 258 estratos. En 2015 la encuesta incluyó 44.202 hogares, distribuidos en 4.739 clúster de 177 estratos. Después de excluir a las mujeres en estado de embarazo y personas con dietas prescritas, fueron analizados 37.981 registros. En 2010; 10.150 entre 5 a 17 años (niños) y 5.145 entre 18 a 64 años (Adultos). En 2015; 13.243 entre 5 y 17 años y 9.443 entre 18 y 64 años.

\section{Fuentes de datos}

En las ENSIN 2010 y 2015, la persona cabeza del hogar suministró información demográfica, socioeconómica, sobre seguridad alimentaria y del nivel de riqueza del hogar. Además, nutricionistas aplicaron un CFC. Los menores de doce años fueron asistidos por sus cuidadores para responder el CFC. Las diez respuestas en el CFC sobre la frecuencia de consumo de 28 ítems de alimentos y dos prácticas de interés en nutrición pública fueron convertidas a una variable continua, "veces/día"15,16. Las dos variables de interés fueron, a) la prevalencia de consumo o de realización de la práctica de interés en nutrición pública y b) la frecuencia/día - "veces/día"- de consumo o de realización de la práctica de cada uno de los 28 ítems del CFC.

La talla fue establecida con estadiómetros (Shorr Producctions LCC, Olney, MD, USA), aproximando al milímetro más cercano. El peso fue establecido con básculas SECA, (modelo 872 en 2010 y modelo 874 en 2015).

Se consideraron otras covariables que fueron utilizadas para ajustar las diferencias en la prevalencia y la frecuencia/día de consumo entre encuestas; el sexo, la edad, la seguridad alimentaria del hogar, el índice de riqueza, la región, la etnia, el nivel de urbanismo, el estado de nutrición (IMC) -en niños para facilitar la comparación internacional el equivalente según $\mathrm{IOTF}^{17}$ - y la educación del jefe del hogar. El nivel de urbanismo fue categorizado como los que viven en áreas urbanas de grandes ciudades y el resto como rural. La seguridad alimentaria del hogar fue establecida con la escala ELCSA que mide el acceso físico a los alimentos y los clasifica como seguros y con inseguridad leve, moderada y severa $^{18}$. La etnia se clasificó como mestizo, indígena y afrodescendientes. La riqueza fue establecida con el índice diseñado para la encuesta internacional de demografía y salud ${ }^{19}$. El índice de riqueza fue categorizado en quintiles. La escolaridad de la cabeza del hogar se clasificó como con menos de primaria, primaria, secundaria y técnica/universitaria.

\section{Análisis estadístico}

Todos los análisis fueron conducidos utilizando las rutinas para diseños muestrales complejos del software Stata, versión $14.1^{20}$. El análisis se condujo para estimar, a) la prevalencia -probabilidad de consumo- $y$ la frecuencia/día de consumo de cada ítem en el CFC $y, b)$ para establecer los cambios alimentarios, para esto último, utilizando regresión lineal múltiple con la probabilidad y la frecuencia/día de consumo como las variables dependientes, se estimaron diferencias ajustadas por las covariables de interés y sus intervalos de confianza del 95\% (IC95\%). Las diferencias ajustadas incorporaron el diseño complejo de la muestra y el modelo de regresión múltiple incluyó las covariables de interés.

\section{Resultados}

\section{Características de la población estudiada}

En 2010, la edad media de la población estudiada fue de 21,1 años (IC 95\%: 20,8 a 21,4), el 43,5\%, fueron hombres, el 35,8\% (IC 95\%: 34,6 a 37,0 ) de los hogares tenían seguridad alimentaria, el $73,8 \%$ de los hogares eran urbanos, el $84,9 \%$ se auto declaró mestizo, el $16,5 \%$ de la población encuestada habitaba en Bogotá (La capital del país), el 24,8\% en la región central, el 21,3\% en la región Atlántica, el 18,6\% en la Oriental y el 16,2\% en la región pacífica. El 40,6\% de los jefes del hogar alcanzó como escolaridad máxima el grado de primaria.

En 2015, la edad media de la población estudiada fue de 28,9 años (IC 95\%: 28,0 a 29,8), el 48,1\%, fueron hombres, el 39,6\% (IC 95\%: 36,3 a 43,0) de los hogares tenían seguridad alimentaria, el $77,2 \%$ de los hogares eran urbanos, el $88,6 \%$ se auto declaró mestizo, el 16,2\% de la población encuestada habitaba en Bogotá, el 25,0\% en la región central, el 22,9\% en la región Atlántica, el $17,6 \%$ en la Oriental y el $15,7 \%$ en la región pacífica. El $68,7 \%$ de los jefes del hogar alcanzo como escolaridad máxima el grado de primaria. Si bien entre encuestas y grupos etarios de interés algunas variables sociodemográficas presentan diferencias estadísticamente significativas, no lo son social o biológicamente. En la Tabla 1, se presentan otras características de la población por grupo de edad. 
Tabla 1. Características de la población estudiada en dos encuestas nacionales de situación nutricional en Colombia (ENSIN), 2010-2015

\begin{tabular}{|c|c|c|c|c|c|}
\hline \multirow{2}{*}{ Variable } & \multicolumn{2}{|c|}{$\begin{array}{c}2010 \\
{[n=10150]}\end{array}$} & \multicolumn{2}{|c|}{$\begin{array}{c}2015 \\
{[n=13243]}\end{array}$} & \multirow[t]{2}{*}{ Valor $\mathbf{p}$} \\
\hline & Xó\% & $\mathbf{E E}$ & Xó \% & $\mathbf{E E}$ & \\
\hline \multicolumn{6}{|l|}{$5-17$ años } \\
\hline Edad & 11,8 & 0,10 & 11,0 & 0,10 & $<0,0001$ \\
\hline Talla (cm) & 144,0 & 0,30 & 141,3 & 0,40 & $<0,0001$ \\
\hline Sexo & & & & & 0,542 \\
\hline Hombre & 51,5 & 0,01 & 51,9 & 0,01 & \\
\hline Mujer & 48,5 & 0.01 & 48,1 & 0,01 & \\
\hline Grupo de edad & & & & & $<0,0001$ \\
\hline $5-11$ & 36,8 & 0,01 & 46,1 & 0,01 & \\
\hline $12-17$ & 63,2 & 0,01 & 53,9 & 0,01 & \\
\hline Índice de masa corporal $\left(\mathrm{kg} / \mathrm{m}^{2}\right)^{\mathrm{a}}$ & & & & & 0,833 \\
\hline $18,0-24,9$ & 82,3 & 0,01 & 82,6 & 0,01 & \\
\hline $25,0-29,9$ & 13,4 & 0,01 & 13,2 & 0,01 & \\
\hline+30 & 4,3 & 0,00 & 4,2 & 0,01 & \\
\hline 18 a 64 años & $\mathrm{n}=5145$ & & $n=9443$ & & \\
\hline Edad & 37,5 & 0,2 & 37,8 & 0,2 & 0,083 \\
\hline Talla (cm) & 160,9 & 0,2 & 161,3 & 0,2 & 0,561 \\
\hline Sexo & & & & & 0,861 \\
\hline Hombre & 44,6 & 0,01 & 46,1 & 0,01 & \\
\hline Mujer & 55,4 & 0,01 & 53,9 & 0,01 & \\
\hline Grupo de edad & & & & & 0,034 \\
\hline $18-24$ & 21,6 & 0,01 & 20,3 & 0,01 & \\
\hline $25-34$ & 24,0 & 0,01 & 24,8 & 0,01 & \\
\hline $35-44$ & 22,0 & 0,01 & 20,9 & 0,01 & \\
\hline $45-54$ & 19,2 & 0,01 & 20,9 & 0,01 & \\
\hline $55-64$ & 13,2 & 0,01 & 12,9 & 0,01 & \\
\hline Índice de masa corporal $\left(\mathrm{kg} / \mathrm{m}^{2}\right)$ & & & & & $<0,0001$ \\
\hline $18,0-24,9$ & 48,3 & 0,01 & 43,7 & 0,01 & \\
\hline $25,0-29,9$ & 36,4 & 0,01 & 37,6 & 0,01 & \\
\hline+30 & 15,3 & 0,01 & 18,7 & 0,01 & \\
\hline Índice de riqueza & & & & & $<0,0001$ \\
\hline Q1 & 16,9 & 0,01 & 9,9 & 0,02 & \\
\hline Q2 & 18,5 & 0,01 & 13,2 & 0,03 & \\
\hline Q3 & 21,2 & 0,01 & 15,0 & 0,02 & \\
\hline Q4 & 21,8 & 0,01 & 24,8 & 0,02 & \\
\hline Q5 & 21,6 & 0,01 & 37,1 & 0,05 & \\
\hline
\end{tabular}

${ }^{\text {a }}$ Basado en los equivalentes de IMC y puntos de corte según la clasificación IOTF ${ }^{17}$

Características del consumo y diferencias entre encuestas [2015 menos 2010]

En los niños, la prevalencia y frecuencia de consumo disminuyó en trece alimentos. La frecuencia/día aumentó en seis alimentos. La prevalencia de consumo de leche, carne, atún, vísceras, leguminosas, pan, tubérculos, azúcar, gaseosas y alimentos fritos disminuyó, al igual que la de verduras y frutas. La frecuencia/día para algunos de éstos también disminuyó, Tablas 2 y 3. 
Tabla 2. Prevalencia alcanzada en el consumo de alimentos y prácticas de interés pública; Diferencias ajustadas [2015-2010] para los niños (5-17 años). Con base en ENSIN-2010 y ENSIN-2015, Colombia.

\begin{tabular}{|c|c|c|c|c|}
\hline \multirow[b]{2}{*}{ Variable } & \multicolumn{2}{|c|}{ Prevalencia (IC 95\%) } & \multirow{2}{*}{$\begin{array}{l}\text { Diferencia Ajustada } \\
\text { (IC 95\%) }\end{array}$} & \multirow[b]{2}{*}{ Valor p } \\
\hline & $\begin{array}{c}2010 \\
{[n=10150]}\end{array}$ & $\begin{array}{c}2015 \\
{[n=13243]}\end{array}$ & & \\
\hline Leche-líquida o polvo & $94,5(93,8$ a 95,1$)$ & $92,4(90,5$ a 94,0$)$ & $-2,77(-4,14$ a $-1,39)$ & $<0,0001$ \\
\hline Queso y lácteos & $86,2(85,1$ a 87,2$)$ & $87,4(85,0$ a 89,4$)$ & $-0,57(-2,73$ a 1,59$)$ & 0,605 \\
\hline Huevos & $96,8(96,3$ a 97,2$)$ & $96,6(95,9$ a 97,1$)$ & $-0,00(-0,01$ a 0,01$)$ & 0,999 \\
\hline Carne de res, ternera... & $95,2(94,6$ a 95,7$)$ & $93,3(91,8$ a 94,5$)$ & $-2,45(-3,68$ a $-1,22)$ & $<0,0001$ \\
\hline Pollo o gallina & $93,3(92,6$ a 94,0$)$ & $94,4(93,1$ a 95,5$)$ & $0,29(-1,16$ a 1,73$)$ & 0,697 \\
\hline Atún o sardinas & $62,2(60,9$ a 63,6$)$ & $58,0(54,8$ a 61,1$)$ & $-5,19(-8,08$ a $-2,31)$ & $<0,0001$ \\
\hline Pescados o mariscos & $59,4(57,9$ a 60,9$)$ & $57,6(53,5$ a 61,6$)$ & $-0,37(-4,12$ a 3,38$)$ & 0,846 \\
\hline Embutidos & $76,9(75,6$ a 78,2$)$ & $78,5(75,1$ a 81,6$)$ & $-0,51(-2,97$ a 1,95$)$ & 0,683 \\
\hline Morcilla o vísceras & $39,0(37,7$ a 40,4$)$ & $31,5(29,6$ a 33,5$)$ & $-6,28(-8,84$ a $-3,73)$ & $<0,0001$ \\
\hline Menudencias de pollo & $47,9(46,4$ a 49,4$)$ & $43,4(39,2$ a 47,6$)$ & $-0,41(-3,92$ a 3,09$)$ & 0,817 \\
\hline Granos secos & $96,6(96,1$ a 97,0$)$ & $94,9(93,7$ a 96,0$)$ & $-1,72(-2,92$ a $-0,52)$ & 0,005 \\
\hline Arroz o pasta & $99,8(99,6$ a 99,9$)$ & $99,6(99,3$ a 99,8$)$ & $-0,16(-0,51$ a 0,20$)$ & 0,388 \\
\hline Pan, arepa o galleta ${ }^{b}$ & $98,6(98,3$ a 98,9$)$ & $92,9(91,2$ a 94,3$)$ & $-5,81(-6,87$ a $-4,75)$ & $<0,0001$ \\
\hline Tubérculos o plátanos & $98,1(97,7$ a 98,4$)$ & $96,3(95,2$ a 97,2$)$ & $-1,49(-2,36 \mathrm{a}-0,61)$ & 0,001 \\
\hline Verduras cocidas & $60,7(59,2$ a 62,3$)$ & $62,8(57,6$ a 67,7$)$ & $1,47(-1,26$ a 4,21$)$ & 0,290 \\
\hline Verduras crudas & $73,8(72,6$ a 74,9$)$ & $71,4(69,3$ a 73,4$)$ & $-1,91(-4,27$ a 0,44$)$ & 0,112 \\
\hline Frutas en jugo & $89,6(88,7$ a 90,4$)$ & $89,5(87,5$ a 91,3$)$ & $-1,21(-3,09$ a 0,67$)$ & 0,208 \\
\hline Frutas enteras & $89,5(88,6$ a 90,4$)$ & $84,3(81,2$ a 87,0$)$ & $-6,46(-8,55$ a $-4,37)$ & $<0,0001$ \\
\hline Comidas rápidas & $52,0(50,3$ a 53,6$)$ & $61,3(54,1$ a 68,0$)$ & $2,37(-0,17$ a 4,90$)$ & 0,067 \\
\hline Mantequilla, crema... & $30,5(29,1$ a 32,0$)$ & $33,7(30,0$ a 37,6$)$ & $2,10(-1,04$ a 5,24$)$ & 0,190 \\
\hline Café o té & $66,9(65,5$ a 68,4$)$ & $60,5(56,3$ a 64,5$)$ & $-3,88(-6,68$ a $-1,08)$ & 0,007 \\
\hline Panela, azúcar o miel & $99,4(99,1$ a 99,5$)$ & $94,7(93,6$ a 95,7$)$ & $-4,97(-6,35$ a $-3,59)$ & $<0,0001$ \\
\hline Gaseosas o refrescos & $85,3(84,3$ a 86,3$)$ & $83,3(81,3$ a 85,2$)$ & $-3,77(-5,73 \mathrm{a}-1,80)$ & $<0,0001$ \\
\hline Alimentos de paquete & $82,4(81,3$ a 83,4$)$ & $82,6(80,5$ a 84,5$)$ & $1,92(-0,14$ a 3,98$)$ & 0,068 \\
\hline Alimentos fritos & $96,6(96,1$ a 97,1$)$ & $93,2(92,2$ a 94,1$)$ & $-3,34(-4,60 \mathrm{a}-2,09)$ & $<0,0001$ \\
\hline Golosinas o dulces & $88,0(87,1$ a 88,9$)$ & $87,7(85,9$ a 89,4$)$ & $-1,77(-3,83$ a 0,30$)$ & 0,093 \\
\hline Alimentos "light" & $5,5(4,9$ a 6,2$)$ & $4,6(3,9$ a 5,4$)$ & $-2,65(-3,92 \mathrm{a}-1,38)$ & $<0,0001$ \\
\hline Alimentos integrales & $17,7(16,5$ a 18,9$)$ & $18,5(15,2$ a 23,3$)$ & $-1,23(-4,04$ a 1,58$)$ & 0,391 \\
\hline Alimentos en la calle & $41,0(39,6$ a 42,5$)$ & $38,8(34,0$ a 43,8$)$ & $-3,64(-6,87$ a $-0,41)$ & 0,027 \\
\hline Usa salero en la mesa & $16,2(15,3$ a 17,3$)$ & $14,2(11,9$ a 16,9$)$ & $-1,90(-4,57$ a 0,77$)$ & 0,164 \\
\hline
\end{tabular}

a Diferencias ajustadas en un modelo de regresión lineal donde $\hat{Y}$ es la prevalencia del ítem de interés, la principal variable explicatoria es el año de la encuesta y, además, las siguientes covariables; el sexo, la edad, el índice de riqueza, la región, la etnia, el nivel de urbanismo y el estado de nutrición (IMC) -En los niños, con base en el índice equivalente recomendado por $\mathrm{IOTF}^{17}$ : Todos los análisis incorporaron el diseño complejo de la muestra. ${ }^{\mathrm{b}}$ En 2015 se separó el ítem y solo se preguntó por Pan. 
Tabla 3. Frecuencia (Veces/día) alcanzada en el consumo de alimentos y prácticas de interés pública; Diferencias ajustadas 2015 2010 para los niños (5-17 años). Con base en ENSIN-2010 y ENSIN-2015, Colombia.

\begin{tabular}{|c|c|c|c|c|}
\hline \multirow{2}{*}{ Variable } & \multicolumn{2}{|c|}{ Frecuencia -Veces/día (IC 95\%) } & \multirow{2}{*}{$\begin{array}{c}\text { Diferencia Ajustada } \\
\text { (IC 95\%) }\end{array}$} & \multirow{2}{*}{ Valor $\mathbf{p}$} \\
\hline & 2010 & 2015 & & \\
\hline Leche-líquida o polvo & $1,07(1,04$ a 1,09$)$ & $0,96(0,85$ a 1,06$)$ & $-0,11(-0,18$ a $-0,04)$ & 0,001 \\
\hline Queso y lácteos & $0,56(0,54$ a 0,58$)$ & $0,58(0,53$ a 0,63$)$ & $0,00(-0,04$ a 0,05$)$ & 0,845 \\
\hline Huevos & $0,69(0,68$ a 0,71$)$ & $0,76(0,71$ a 0,80$)$ & $0,07(0,03$ a 0,11$)$ & 0,002 \\
\hline Carne de res, ternera... & $0,57(0,55$ a 0,58$)$ & $0,49(0,46$ a 0,52$)$ & $-0,10(-0,14$ a $-0,06)$ & $<0,0001$ \\
\hline Pollo o gallina & $0,35(0,34$ a 0,36$)$ & $0,39(0,36$ a 0,41$)$ & $0,04(0,01$ a 0,06$)$ & 0,004 \\
\hline Atún o sardinas & $0,15(0,15$ a 0,16$)$ & $0,18(0,16$ a 0,19$)$ & $0,03(0,01$ a 0,05$)$ & $<0,0001$ \\
\hline Pescados o mariscos & $0,20(0,19$ a 0,22$)$ & $0,21(0,18$ a 0,24$)$ & $0,02(-0,01$ a 0,05$)$ & 0,274 \\
\hline Embutidos & $0,42(0,40$ a 0,43$)$ & $0,43(0,40$ a 0,46$)$ & $0,01(-0,02$ a 0,05$)$ & 0,452 \\
\hline Morcilla o vísceras & $0,14(0,13$ a 0,14$)$ & $0,15(0,13$ a 0,17$)$ & $0,03(0,00$ a 0,05$)$ & 0,023 \\
\hline Menudencias de pollo & $0,23(0,21$ a 0,24$)$ & $0,23(0,21$ a 0,25$)$ & $0,02(-0,01$ a 0,04$)$ & 0,169 \\
\hline Granos secos & $0,58(0,56$ a 0,59$)$ & $0,46(0,44$ a 0,49$)$ & $-0,09(-0,13$ a $-0,06)$ & $<0,0001$ \\
\hline Arroz o pasta & $1,85(1,82$ a 1,87$)$ & $1,69(1,62$ a 1,75$)$ & $-0,16(-0,23$ a $-0,09)$ & $<0,0001$ \\
\hline Pan, arepa o galleta ${ }^{a}$ & $1,39(1,37$ a 1,42$)$ & $0,94(0,88$ a 1,00$)$ & $-0,42(-0,48$ a $-0,36)$ & $<0,0001$ \\
\hline Tubérculos o plátanos & $1,30(1,28$ a 1,33$)$ & $0,95(0,89$ a 1,00$)$ & $-0,29(-0,35$ a $-0,23)$ & $<0,0001$ \\
\hline Verduras cocidas & $0,44(0,42$ a 0,45$)$ & $0,53(0,50$ a 0,56$)$ & $0,10(0,07$ a 0,13$)$ & $<0,0001$ \\
\hline Verduras crudas & $0,51(0,01$ a 0,53$)$ & $0,54(0,51$ a 0,57$)$ & $0,02(-0,01$ a 0,05$)$ & 0,120 \\
\hline Frutas en jugo & $1,28(1,24$ a 1,30$)$ & $1,15(1,06$ a 1,23$)$ & $-0,19(-0,26$ a $-0,12)$ & $<0,0001$ \\
\hline Frutas enteras & $0,80(0,77$ a 0,82$)$ & $0,68(0,63$ a 0,74$)$ & $-0,12(-0,17$ a $-0,07)$ & $<0,0001$ \\
\hline Comidas rápidas & $0,18(0,17$ a 0,18$)$ & $0,18(0,17$ a 0,19$)$ & $0,00(-0,02$ a 0,02$)$ & 0,953 \\
\hline Mantequilla, crema... & $0,52(0,49$ a 0,55$)$ & $0,56(0,49$ a 0,63$)$ & $0,02(-0,03$ a 0,07$)$ & 0,457 \\
\hline Café o té & $1,02(0,99$ a 1,05$)$ & $0,92(0,83$ a 1,00$)$ & $-0,07(-0,15$ a 0,00$)$ & 0,064 \\
\hline Panela, azúcar o miel & $2,58(2,55$ a 2,61$)$ & $1,90(1,81$ a 1,99$)$ & $-0,67(-0,76$ a $-0,59)$ & $<0,0001$ \\
\hline Gaseosas o refrescos & $0,71(0,69$ a 0,74$)$ & $0,71(0,67$ a 0,76$)$ & $-0,02(-0,07$ a 0,03$)$ & 0,405 \\
\hline Alimentos de paquete & $0,66(0,64$ a 0,68$)$ & $0,58(0,54$ a 0,61$)$ & $-0,12(-0,16$ a $-0,08)$ & $<0,0001$ \\
\hline Alimentos fritos & $0,83(0,80$ a 0,85$)$ & $0,65(0,61$ a 0,69$)$ & $-0,18(-0,23$ a $-0,14)$ & $<0,0001$ \\
\hline Golosinas o dulces & $1,20(1,17$ a 1,23$)$ & $1,11(1,05$ a 1,16$)$ & $-0,15(-0,21$ a $-0,08)$ & $<0,0001$ \\
\hline Alimentos "light" & $0,73(0,63$ a 0,83$)$ & $0,50(0,45$ a 0,56$)$ & $-0,24(-0,38$ a $-0,11)$ & 0,001 \\
\hline Alimentos integrales & $0,49(0,46$ a 0,52$)$ & $0,54(0,51$ a 0,58$)$ & $0,09(0,03$ a 0,14$)$ & 0,002 \\
\hline Alimentos en la calle & $0,30(0,28$ a 0,31$)$ & $0,35(0,32$ a 0,38$)$ & $0,07(0,03$ a 0,10$)$ & $<0,0001$ \\
\hline Usa salero en la mesa & $0,02(0,02$ a 0,02$)$ & $0,88(0,81$ a 0,96$)$ & $0,87(0,78$ a 0,95$)$ & $<0,0001$ \\
\hline
\end{tabular}

${ }^{a}$ Diferencias ajustadas en un modelo de regresión lineal donde $\hat{Y}$ es la prevalencia del ítem de interés, la principal variable expiatoria es el año de la encuesta y, además, las siguientes covariables; el sexo, la edad, el índice de riqueza, la región, la etnia, el nivel de urbanismo y el estado de nutrición (IMC) -En los niños, con base en el índice equivalente recomendado por IOFT ${ }^{17}$ : Todos los análisis incorporaron el diseño complejo de la muestra. ${ }^{b}$ En 2015 se separó el ítem y solo se preguntó por Pan.

En los adultos, la prevalencia de consumo disminuyó en once alimentos y aumentó en dos. La frecuencia de consumo disminuyó en quince alimentos y aumentó 26 en dos. La prevalencia de leche, carne, atún, vísceras, pan, tubérculos, azúcar, alimentos fritos y alimentos light disminuyó, al igual que la de verduras y frutas. En 
niños y adultos la prevalencia de consumo de alimentos de paquete aumentó, pero su frecuencia/día disminuyó, Tablas 4 y 5. La prevalencia de consumo de alimentos en la calle disminuyó en los niños, pero no en los adultos y la frecuencia/día aumentó en ambos. La prevalencia de uso del salero disminuyó en niños y adultos, pero su frecuencia/día aumentó.

Tabla 4 Prevalencia alcanzada en el consumo de alimentos y prácticas de interés pública; Diferencias ajustadas [2015-2010] para los adultos (18-64 años). Con base en ENSIN-2010 y ENSIN-2015, Colombia.

\begin{tabular}{|c|c|c|c|c|}
\hline \multirow[b]{2}{*}{ Variable } & \multicolumn{2}{|c|}{ Prevalencia (IC 95\%) } & \multirow{2}{*}{$\begin{array}{c}\text { Diferencia Ajustada }{ }^{\mathrm{a}} \\
\text { (IC } 95 \%)\end{array}$} & \multirow[b]{2}{*}{ Valor $\mathbf{p}$} \\
\hline & $\begin{array}{c}2010 \\
{[n=5145]}\end{array}$ & $\begin{array}{c}2015 \\
{[n=9443]}\end{array}$ & & \\
\hline Leche-líquida o polvo & $90,7(89,7$ a 91,7$)$ & $86,8(84,4$ a 88,8$)$ & $-4,79(-6,74$ a $-2,84)$ & $<0,0001$ \\
\hline Queso y lácteos & $82,4(81,0$ a 83,7$)$ & $82,9(80,7$ a 84,8$)$ & $-0,97(-0,33$ a 1,33$)$ & 0,408 \\
\hline Huevos & $95,4(94,7$ a 96,1$)$ & $95,4(94,4$ a 96,2$)$ & $-0,78(-1,83$ a 2,84$)$ & 0,151 \\
\hline Carne de res, ternera... & $96,0(95,2$ a 96,6$)$ & $94,2(93,4$ a 95,0$)$ & $-2,30(-3,51$ a $-1,10)$ & $<0,0001$ \\
\hline Pollo o gallina & $94,2(93,3$ a 95,0$)$ & $96,0(95,0$ a 96,8$)$ & $1,22(0,00$ a 2,36$)$ & 0,037 \\
\hline Atún o sardinas & $65,5(63,8$ a 67,3$)$ & $62,8(59,6$ a 65,7$)$ & $-4,42(-7,42$ a $-1,41)$ & 0,004 \\
\hline Pescados o mariscos & $64,8(62,9$ a 66,7$)$ & $65,6(61,4$ a 69,5$)$ & $0,52(-3,52$ a 4,55$)$ & 0,801 \\
\hline Embutidos & $64,1(62,3$ a 65,9$)$ & $66,3(63,1$ a 69,4$)$ & $-0,32(-2,64$ a 2,01$)$ & 0,790 \\
\hline Morcilla o vísceras & $46,4(44,6$ a 48,3$)$ & $39,7(37,7$ a 41,8$)$ & $-6,92(-9,92$ a $-3,92)$ & $<0,0001$ \\
\hline Menudencias de pollo & $44,1(42,2$ a 46,0$)$ & $40,5(36,4$ a 44,6$)$ & $-0,57(-3,74$ a 2,60$)$ & 0,725 \\
\hline Granos secos & $94,9(94,0$ a 95,7$)$ & $93,6(92,4$ a 94,6$)$ & $-1,02(-2,42$ a 0,39$)$ & 0,157 \\
\hline Arroz o pasta & $99,5(99,1$ a 99,7$)$ & $99,2(98,6$ a 99,6$)$ & $0,00(-0,36$ a 0,56$)$ & 0,678 \\
\hline Pan, arepa o galleta ${ }^{b}$ & $97,5(96,9$ a 98,0$)$ & $88,4(86,1$ a 90,3$)$ & $-9,97(-11,98$ a $-7,96)$ & $<0,0001$ \\
\hline Tubérculos o plátanos & $98,7(98,2$ a 99,1$)$ & $97,6(96,7$ a 98,3$)$ & $-1,34(-2,36$ a $-0,32)$ & 0,010 \\
\hline Verduras cocidas & $72,6(70,8$ a 74,4$)$ & $73,0(68,9$ a 76,8$)$ & $-1,76(-4,51$ a 0,99$)$ & 0,209 \\
\hline Verduras crudas & $82,9(81,6$ a 84,2$)$ & $80,5(79,0$ a 82,0$)$ & $-3,37(-5,44$ a $-1,30)$ & 0,001 \\
\hline Frutas en jugo & $88,7(87,4$ a 89,8$)$ & $89,6(87,9$ a 91,1$)$ & $0,60(-1,02$ a 2,22$)$ & 0,469 \\
\hline Frutas enteras & $83,2(80,9$ a 83,7$)$ & $76,1(74,3$ a 77,8$)$ & $-7,40(-9,60$ a $-5,20)$ & $<0,0001$ \\
\hline Comidas rápidas & $42,9(40,9$ a 44,8$)$ & $54,6(49,9$ a 59,1$)$ & $7,25(4,73$ a 9,78$)$ & $<0,0001$ \\
\hline Mantequilla, crema... & $28,3(26,6$ a 30,1$)$ & $32,0(26,9$ a 37,5$)$ & $0,42(-2,99$ a 3,84$)$ & 0,808 \\
\hline Café o té & $80,4(79,0$ a 81,8$)$ & $78,1(74,7$ a 81,1$)$ & $-1,97(-4,19$ a 0,25$)$ & 0,082 \\
\hline Panela, azúcar o miel & $97,3(96,6$ a 97,9$)$ & $93,5(92,2$ a 94,5$)$ & $-3,43(-4,58$ a $-2,28)$ & $<0,0001$ \\
\hline Gaseosas o refrescos & $72,5(70,8$ a 74,1$)$ & $71,6(69,6$ a 73,6$)$ & $-1,32(-3,94$ a 1,30$)$ & 0,324 \\
\hline Alimentos de paquete & $43,6(41,8$ a 45,4$)$ & $51,2(49,5$ a 53,0$)$ & $6,73(4,36$ a 9,10$)$ & $<0,0001$ \\
\hline Alimentos fritos & $92,7(91,7$ a 93,5$)$ & $86,5(85,0$ a 87,9$)$ & $-6,06(-7,85$ a $-4,27)$ & $<0,0001$ \\
\hline Golosinas o dulces & $53,9(52,0$ a 55,7$)$ & $54,5(52,8$ a 56,1$)$ & $-0,99(-3,46$ a 1,48$)$ & 0,432 \\
\hline Alimentos "light" & $9,2(8,2$ a 10,4$)$ & $8,8(6,9$ a 11,2$)$ & $-2,29(-3,65$ a $-0,93)$ & 0,001 \\
\hline Alimentos integrales & $22,1(20,5$ a 23,8$)$ & $24,5(20,9$ a 28,5$)$ & $-0,30(-2,61$ a 2,00$)$ & 0,798 \\
\hline Alimentos en la calle & $37,3(35,4$ a 39,1$)$ & $40,4(37,2$ a 43,6$)$ & $1,58(-1,17$ a 4,32$)$ & 0.261 \\
\hline Usa salero en la mesa & $18,5(17,1$ a 20,0$)$ & $17,4(14,1$ a 21,3$)$ & $-2,51(-4,82$ a $-0,19)$ & 0,034 \\
\hline
\end{tabular}

${ }^{a}$ Diferencias ajustadas en un modelo de regresión lineal donde $\hat{Y}$ es la prevalencia del ítem de interés, la principal variable explicatoria es el año de la encuesta y, además, las siguientes covariables; el sexo, la edad, el índice de riqueza, la región, la etnia, el nivel de urbanismo y el estado de nutrición (IMC) y el nivel de escolaridad. Todos los análisis incorporaron el diseño complejo de la muestra. ${ }^{b}$ En 2015 se separó el ítem y solo se preguntó por Pan. 
Tabla 5 Frecuencia (Veces/día) alcanzada en el consumo de alimentos y prácticas de interés pública; Diferencias ajustadas 2015 2010 para los adultos (18-64 años). Con base en ENSIN-2010 y ENSIN-2015, Colombia.

\begin{tabular}{|c|c|c|c|c|}
\hline \multirow{2}{*}{ Variable } & \multicolumn{2}{|c|}{ Frecuencia -Veces/día (IC 95\%) } & \multirow{2}{*}{$\begin{array}{c}\text { Diferencia Ajustada }^{a} \\
\% \text { (IC 95\%) }\end{array}$} & \multirow{2}{*}{ Valor $\mathbf{p}$} \\
\hline & 2010 & 2015 & & \\
\hline Leche-líquida o polvo & $0,95(0,91$ a 0,98$)$ & $0,84(0,75$ a 0,93$)$ & $-0,13(-0,19$ a $-0,07)$ & $<0,0001$ \\
\hline Queso y lácteos & $0,48(0,46$ a 0,50$)$ & $0,48(0,44$ a 0,52$)$ & $-0,02(-0,05$ a 0,01$)$ & 0,172 \\
\hline Huevos & $0,62(0,60$ a 0,64$)$ & $0,69(0,65$ a 0,73$)$ & $0,05(0,01$ a 0,09$)$ & 0,008 \\
\hline Carne de res, ternera... & $0,60(0,58$ a 0,62$)$ & $0,54(0,49$ a 0,58$)$ & $-0,08(-0,12$ a $-0,04)$ & $<0,0001$ \\
\hline Pollo o gallina & $0,37(0,36$ a 0,39$)$ & $0,39(0,36$ a 0,43$)$ & $0,02(-0,01$ a 0,04$)$ & 0,141 \\
\hline Atún o sardinas & $0,15(0,14$ a 0,16$)$ & $0,16(0,15$ a 0,17$)$ & $0,01(-0,00$ a 0,02$)$ & 0,106 \\
\hline Pescados o mariscos & $0,20(0,19$ a 0,21$)$ & $0,21(0,18$ a 0,24$)$ & $0,02(-0,00$ a 0,05$)$ & 0,102 \\
\hline Embutidos & $0,32(0,31$ a 0,34$)$ & $0,34(0,31$ a 0,38$)$ & $0,02(-0,01$ a 0,05$)$ & 0,226 \\
\hline Morcilla o vísceras & $0,12(0,11$ a 0,13$)$ & $0,13(0,11$ a 0,15$)$ & $0,01(-0,00$ a 0,02$)$ & 0,115 \\
\hline Menudencias de pollo & $0,19(0,18$ a 0,21$)$ & $0,20(0,18$ a 0,21$)$ & $0,01(-0,01$ a 0,02$)$ & 0,400 \\
\hline Granos secos & $0,53(0,51$ a 0,55$)$ & $0,43(0,41$ a 0,45$)$ & $-0,10(-0,13$ a $-0,07)$ & $<0,0001$ \\
\hline Arroz o pasta & $1,71(1,69$ a 1,74$)$ & $1,58(1,53$ a 1,63$)$ & $-0,08(-0,15$ a $-0,02)$ & 0,013 \\
\hline Pan, arepa o galleta ${ }^{b}$ & $1,23(1,20$ a 1,26$)$ & $0,84(0,76$ a 0,92$)$ & $-0,40(-0,44$ a $-0,35)$ & $<0,0001$ \\
\hline Tubérculos o plátanos & $1,30(1,27$ a 1,34$)$ & $0,94(0,89$ a 0,98$)$ & $-0,35(-0,40$ a $-0,29)$ & $<0,0001$ \\
\hline Verduras cocidas & $0,48(0,46$ a 0,50$)$ & $0,53(0,49$ a 0,56$)$ & $0,05(0,01$ a 0,09$)$ & 0,006 \\
\hline Verduras crudas & $0,57(0,55$ a 0,59$)$ & $0,61(0,59$ a 0,63$)$ & $0,02(-0,00$ a 0,05$)$ & 0,086 \\
\hline Frutas en jugo & $1,19(1,16$ a 1,23$)$ & $1,11(1,03$ a 1,18$)$ & $-0,13(-0,21$ a $-0,05)$ & 0,003 \\
\hline Frutas enteras & $0,65(0,63$ a 0,68$)$ & $0,63(0,60$ a 0,66$)$ & $-0,05(-0,10$ a $-0,00)$ & 0,040 \\
\hline Comidas rápidas & $0,17(0,15$ a 0,19$)$ & $0,15(0,13$ a 0,16$)$ & $-0,03(-0,05$ a $-0,00)$ & 0,030 \\
\hline Mantequilla, crema... & $0,52(0,48$ a 0,57$)$ & $0,55(0,47$ a 0,63$)$ & $0,01(-0,07$ a 0,09$)$ & 0,796 \\
\hline Café o té & $1,76(1,71$ a 1,80$)$ & $1,52(1,47$ a 1,57$)$ & $-0,24(-0,32$ a $-0,17)$ & $<0,0001$ \\
\hline Panela, azúcar o miel & $2,65(2,61$ a 2,68$)$ & $2,00(1,90$ a 2,09$)$ & $-0,61(-0,70$ a $-0,52)$ & $<0,0001$ \\
\hline Gaseosas o refrescos & $0,70(0,66$ a 0,74$)$ & $0,66(0,63$ a 0,70$)$ & $-0,05(-0,10$ a 0,00$)$ & 0,055 \\
\hline Alimentos de paquete & $0,38(0,35$ a 0,40$)$ & $0,29(0,27$ a 0,31$)$ & $-0,09(-0,12$ a $-0,05)$ & $<0,0001$ \\
\hline Alimentos fritos & $0,74(0,71$ a 0,77$)$ & $0,58(0,52$ a 0,63$)$ & $-0,16(-0,20 \mathrm{a}-0,11)$ & $<0,0001$ \\
\hline Golosinas o dulces & $0,79(0,74$ a 0,83$)$ & $0,62(0,60$ a 0,65$)$ & $-0,20(-0,26$ a $-0,14)$ & $<0,0001$ \\
\hline Alimentos "light" & $0,74(0,63$ a 0,84$)$ & $0,53(0,43$ a 0,63$)$ & $-0,21(-0,36$ a $-0,05)$ & 0,008 \\
\hline Alimentos integrales & $0,58(0,53$ a 0,63$)$ & $0,59(0,55$ a 0,64$)$ & $-0,13(-0,09$ a 0,06$)$ & 0,729 \\
\hline Alimentos en la calle & $0,11(0,10$ a 0,12$)$ & $0,33(0,30$ a 0,35$)$ & $0,05(0,01$ a 0,08$)$ & 0,009 \\
\hline Usa salero en la mesa & $0,02(0,02$ a 0,02$)$ & $0,85(0,79$ a 0,91$)$ & $0,84(0,76$ a 0,91$)$ & $<0,0001$ \\
\hline
\end{tabular}

${ }^{a}$ Diferencias ajustadas en un modelo de regresión lineal donde $\hat{Y}$ es la frecuencia/día del ítem de interés, la principal variable explicatoria es el año de la encuesta y, además, las siguientes covariables; el sexo, la edad, el índice de riqueza, la región, la etnia, el nivel de urbanismo y el estado de nutrición (IMC) y el nivel de escolaridad. Todos los análisis incorporaron el diseño complejo de la muestra. ${ }^{\mathrm{b}}$ En 2015 se separó el ítem y solo se preguntó por Pan.

\section{Conclusiones}

El cambio alimentario establecido para el quinquenio 2010-2015 es similar en niños y adultos, confirmando que la cultura alimentaria se hereda socialmente y se transmite de manera vertical entre padres e hijos ${ }^{21}$, lo que justificaría intervenciones en adultos y niños, no sólo en estos últimos. 


\section{Cambios en la prevalencia y frecuencia/día del consumo usual de alimentos}

La disminución en niños y adultos tanto en la prevalencia y frecuencia/día de consumo de leche, carnes, atún, vísceras y leguminosas secas -alimentos fuentes de proteínas- no es compensada por el leve aumento en la frecuencia/día del consumo de huevos, pollo y gallina. Análisis no publicados basados en la ENSIN-2005, donde la fuente de datos del consumo fue un recordatorio de las últimas 24 horas mostraron ingesta de proteínas por encima de las recomendaciones poblacionales en todos los grupos etarios. Un estudio realizado en 2009 en una muestra independiente ratificó este hallazgo ${ }^{22}$. Explicar por qué disminuyó el consumo de alimentos, fuente de proteínas o en general de cualquier alimento del CFC aplicado, escapa al alcance del estudio. Sin embargo y a manera de hipótesis, es plausible que, a pesar del crecimiento económico del país, la inequidad ${ }^{23,24}$ y el aumento de los impuestos directos e indirectos en los estratos socioeconómicos medio e incluso en el bajo ${ }^{25,26}$, contrajo el gasto en alimentos -al menos en los más costosos-, como son los alimentos fuente de proteínas. En Colombia existen profundas desigualdades e inequidades, es uno de los países con más impuestos sobre las ganancias y cada vez más, los pagan los asalariados, las capas medias y las bajas de la escala socioeconómica; en 2013 ocupaba el puesto 137 entre 140 por su baja competitividad ${ }^{27}$. Estos hallazgos contradicen lo que se ha reportado para la región, en las Américas, en los últimos cuarenta años ha sido notable el descenso en el aporte a la energía total/día que hacen los carbohidratos, a expensas del aumento en el aporte que hace la ingesta de proteína animal, leche carne y huevos principalmente ${ }^{6,28,29}$. Además, el reemplazo de la grasa animal por la vegetal y de los alimentos básicos o tradicionales con poco o ningún procesamiento por los mismos $\mathrm{u}$ otros, pero con alto nivel de procesamiento industrial $1^{6,28,30-32}$. Más recientemente, se ha notado la disminución en el consumo de alimentos fritos, empaquetados, de azúcar y como resultado de la tendencia hacia la "dieta saludable", el incremento del consumo de alimentos bajos en calorías "ligth", de frutas y verduras o con alto contenido de fibra "integrales" 29,33 . Sin embargo, los datos reportados para la región no representan a los países de América Latina de ingresos bajos y medios como Colombia, pues es en éstos precisamente por los costos, que no se realizan estudios poblacionales y representativos del consumo dietario. Lo anterior también dificulta la comparación de estos resultados.
La disminución en la prevalencia y frecuencia/día en el consumo de pan, tubérculos, gaseosas, alimentos fritos, azúcar, panela y miel es deseable y se explicaría como hipótesis, por la incorporación en la decisión de compra y consumo de los mensajes sobre disminuir el consumo snack o los alimentos densos en energía, que reiterativamente se reciben a través de los medios de comunicación o programas de educación por parte de los profesionales en salud y $\mathrm{ONG}^{\prime} \mathrm{s}^{34}$. A pesar de que en Colombia no hay política pública sobre el consumo de este tipo de alimentos, las campañas en contra del consumo de alimentos fritos, densos en carbohidratos complejos -en el imaginario de los sujetos "harinas", de azúcar, de gaseosas y bebidas azucaradas y ultra procesados-, sumadas a los intentos por grabar el consumo de algunos de estos alimentos, han llevado a un imaginario de "dieta saludable" si se evita este tipo de consumo. Como ya se había afirmado, el contexto donde ocurre el consumo es determinante del mismo, los conceptos de "vecindario obeso génico", de "escuela saludable" o de "sellos de advertencia" en el etiquetado, son consecuencia de lo anterior.

Si bien en general hubo cambios deseables, en los adultos también aumentó la prevalencia y frecuencia/día del consumo usual de las comidas rápidas y de paquete. Complementariamente se evidenció disminución del consumo de verduras crudas, de frutas enteras y en jugo en niños y adultos. Consumir frutas o verduras es costoso, para su conservación se necesita de cadena de frío e incorporarlas a la dieta es complejo y, además, requiere de transformaciones en la cultura alimentaria ${ }^{35}$.

\section{Sobre las prácticas de interés en nutrición pública}

Si bien el uso del salero es un proximal de la ingesta de sodio, aquí no se estableció la cantidad consumida. Siempre será deseable disminuir el uso del salero y el consumo de sodio visible e invisible, por la relación con la enfermedad hipertensiva ${ }^{36}$. Ahora es necesario tipificar al sujeto que disminuye su consumo de alimentos "no deseables", pero aumenta las prácticas "de riesgo" como consumir comida callejera, para entender si lo hace como parte de un proceso planificado o sólo en alimentos o prácticas específicas de manera coyuntural o aleatoria. Esta contradicción ilustra lo complejo del comportamiento alimentario de los sujetos. Comer en la calle es una expresión de la modernidad y de las nuevas formas en que los individuos se articulan al aparato productivo, pero en condiciones de higiene no controlada como es la norma en Colombia, se convierte en un riesgo real 
de morbimortalidad por toxiinfecciones alimentarias, incapacidades médicas con la consecuente pérdida de productividad y parasitosis que llevan a anemia, otro de los problemas de nutrición pblica en Colombia ${ }^{13,14}$.

\section{Alcance y limitaciones de estudio}

La principal fortaleza de este estudio es que los datos que dan origen al mismo fueron recolectados en dos ENSIN y tienen representatividad nacional. El CFC aplicado es un instrumento validado y una metodología útil en la aproximación del consumo dietario y de las prácticas de interés en nutrición y salud pública ${ }^{1,13-16}$. Además, fue aplicado por nutricionistas-dietistas entrenados. Como limitaciones se debe mencionar el posible sesgo de información sobre los ítems más sensibles, como el consumo de bebidas azucaradas incluidas las gaseosas, o los alimentos de paquete o sobre otros que son socialmente valorados por considerarse como "el deber ser" por ejemplo, el consumo de frutas o de verduras. Sin embargo, los resultados no sugieren subestimación o sobreestimación de éstos. Finalmente, la Tabla 1 evidencia que la muestra de niños en la ENSIN-2015 tuvo una estructura de edad diferente a la ENSIN-2010. Además, que en 2015 la muestra de adultos tenía mejor condición socioeconómica según el índice de riqueza. Estos dos aspectos que pueden generar confusión sobre los resultados presentados se corrigen en buena medida como se hizo aquí, al ajustar las diferencias en modelos multivariados.

\section{Consideraciones éticas}

La investigación se realizó acorde con la Declaración de Helsinki. El consentimiento verbal fue obtenido en las ENSIN previo al reclutamiento de los hogares y sujetos por el Instituto Colombiano de Bienestar Familiar. Las bases de datos que dieron origen a estos análisis están anonimizadas y disponibles para el público bajo solicitud razonada al Ministerio de Salud de Colombia.

\section{Conflicto de interés}

Los autores declaramos que no tenemos conflictos de interés reales o potenciales sobre el trabajo desarrollado o los resultados alcanzados.

\section{Referencias}

1. Willett W. Nutritional epidemiology. Third Ed. New York: Oxford University Press; 2013. p. 71-141.

2. Satija A, Stampfer M, Rimm M, Willet W, Hu F. Perspective: Are Large, Simple Trials the Solution for Nutrition Research? Adv Nutr. 2018; 9(4): 378387. doi: 10.1093/advances/nmy030.

3. Herrán ÓF, Patiño GA, Del Castillo SE. Dietary transition and excess weight in adults according to the Encuesta de la situación nutricional en Colombia, 2010. Biomédica. 2016; 36(1): 109-120. doi: 10.7705/biomedica.v36i1.2579.

4. Popkin BM, Adair LS, Ng SW. Global nutrition transition and the pandemic of obesity in developing countries. Nutr Rev. 2012; 70(1): 3-21. doi: 10.1111/j.1753-4887.2011.00456.x.

5. Popkin BM. The shift in stages of the nutrition transition in the developing world differs from past experiences! Public Health Nutr. 2002; 5(1A): 205-214.

6. Kearney J. Food consumption trends and drivers. Philos Trans R Soc Lond B Biol Sci. 2010; 365(1554): 2793-2807. doi: 10.1098/rstb.2010.0149.

7. Popkin BM. Nutrition in transition: The changing global nutrition challenge. Asia Pac J Clin Nutr. 2001; (10 Suppl): S13-8.

8. McDonald CM, Baylin A, Arsenault JE, MoraPlazas M, Villamor E. Overweight is more prevalent than stunting and is associated with socioeconomic status, maternal obesity, and a snacking dietary pattern in school children from Bogota, Colombia. J Nutr. 2009; 139(2): 370-376. doi: 10.3945/ jn.108.098111.

9. Babu SC, Gajanan SN, Sanyal P. Changes in food consumption patterns: its importance to food security - application of one-way ANOVA. In: Food Security, Poverty and Nutrition Policy Analysis. Second Ed. New York: Elsevier; 2014. p. 117-138.

10. Almas R, Campbell H, editors. Rethinking agricultural policy regimes: food security, climate change and the future resilience of global agriculture. Vol. 18. New York. Eemeraldinsight; 2012. p. 304.

11. Schmidhuber J. The growing global obesity problem: some policy options to address it. eJADE: Elect J Agricult Develop Econ. 2004; 1(2) : 272-290.

12. White J, Climate Change Agriculture and Food Security (CGIAR), Research Program (CCAFS). Changing our food consumption patterns is necessary to slow climate change, but is largescale change feasible? | CCAFS: CGIAR research program on Climate Change, Agriculture and Food Security. 2016.

13. Instituto Colombiano de Bienestar Familiar. Resumen Ejecutivo Encuesta Nacional de la Situación Nutricional en Colombia, ENSIN 2010. 
Bogotá: ICBF; 2011. 512 p.

14. Profamilia, Instituto Nacional de Salud, Universidad de Antioquia, OPS, Instituto Colombiano de Bienestar Familiar. Encuesta Nacional de la Situación Nutricional de Colombia, ENSIN, 2005. Bogotá: ICBF; 2006. p. 465.

15. Bautista LE, Herrán OF, Pryer JA. Development and simulated validation of a food-frequency questionnaire for the Colombian population. Public Health Nutr. 2005; 8(2): 181-188. doi: 10.1079/ phn2004672.

16. Herrán OF, Ardila MF. Validity and reproducibility of two semi-quantitative alcohol frequency questionnaires for the Colombian population. Public Health Nutr. 2006; 9(6): 763-770. doi: 10.1079/ phn2005880.

17. Cole TJ, Lobstein T. Extended international (IOTF) body mass index cut-offs for thinness, overweight and obesity. Pediatr Obes. 2012; 7(4): 284-294. doi: 10.1111/j.2047-6310.2012.00064.x.

18. FAO. Escala latinoamericana y caribeña de seguridad alimentaria (ELCSA) - manual de uso y aplicación, comité científico de la ELCSA, 2012.

19. Rutstein SO. The DHS Wealth Index: Approaches for rural and urban areas. Demographic and Health Research. Calverton, Maryland, Macro International, MEASURE DHS, 2008. p. (DHS Working Papers No. 60). http://www.popline.org/ node/209748.

20. StataCorp. Stata Statistical Software: Release 14. College Station, TX: StataCorp LP. 2015.

21. Ardila VA, Prada GE, Herrán OF. Distribución de energía y macronutrientes en hogares colombianos. Biomédica. 2013; 33: 163-174. doi: http://dx.doi. org/10.7705/biomedica.v33i2.1451.

22. Herrán O, Del Castillo S, Patiño GA. Exceso de proteínas en la pobreza: la paradoja del exceso de peso en niños colombianos. Rev Chil Nutr. 2017; 44(1): 45-56. doi: doi.org/10.4067/S071775182017000100007 .

23. Departamento Administrativo Nacional de Estadística (DANE). Pobreza monetaria y desigualdad (Resumen). Bogotá, D.C.; 2015. https://www.dane.gov.co/files/investigaciones/ condiciones_vida/pobreza/bol_pobreza_15_pdf.

24. OPS. Peña M, Ballacao J (Editors). La obesidad en la pobreza: un nuevo reto para la salud pública. Publicación científica No. 576. Washington, DC.: OPS; 2000.

25. Zapata JG, Ariza N. Eficiencia de la política tributaria y su relación con el gasto público en la comunidad Andina; el caso de Colombia. Coyuntura
Soc. 2005; 33:103-128.

26. López HF, Chaparro MS. Equidad y ética en el recaudo tributario colombiano: 1990-2010. Apuntes del CENES. 2014; 33(58): 83-108. doi: https://doi. org/10.19053/22565779.3101.

27. World Bank/international finance corporation; doing business 2015: paying taxes; date of data collection or release: 20th November 2014; www. doingbusiness.org. Global Competitiveness Report 2015-2016 - Reports - World Economic Forum. http://reports.weforum.org/global-competitivenessreport-2015.

28. de Oliveira SP. Changes in food consumption in Brazil. Arch Latinoam Nutr. 1997; 47(2 Suppl 1): $22-24$.

29.FAO. Global and regional food consumption patterns and trends. http://www.fao.org/docrep/005/ ac911e/ac911e05.htm.

30. Nasreddine L, Tamim H, Itani L, Nasrallah MP, Isma'eel $\mathrm{H}$, Nakhoul $\mathrm{NF}$, et al. A minimally processed dietary pattern is associated with lower odds of metabolic syndrome among Lebanese adults. Public Health Nutr. 2018; 21(1): 160-171. doi: $10.1017 /$ S1368980017002130.

31. Crovveto M, Uauy R. Evolución del gasto en alimentos procesados en la población del Gran Santiago en los últimos 20 años. Rev Med Chil. 2012; 140(3): 305-312. doi: doi.org/10.4067/S003498872012000300004.

32. OPS, OMS. Alimentos y bebidas ultraprocesados en América Latina: tendencias, efecto sobre la obesidad e implicaciones para las políticas públicas. Wasington: OPS. OMS; 2015. p. 74.

33. Bleich SN, Wolfson JA. Trends in SSBs and snack consumption among children by age, body weight, and race/ethnicity. Obesity. 2015; 23(5): 1039-1046. doi: 10.1002/oby.21050.

34. Harris JL, Bargh JA. Television viewing and unhealthy diet: implications for children and media interventions. Health Commun. 2009; 24(7): 660673. doi: 10.1080/10410230903242267.

35. Prada GE, Herrán OF. Impact of strategies to increase the consumption of fruits and vegetables in Colombia. Revi Chil Nutr. 2009; 36(4): 1080-1089. doi: doi.org/10.4067/S071775182009000400004 .

36. Minsalud, Colombia. Estrategia nacional para la reducción del consumo de sal/sodio en Colombia 2012 - 2021. Bogotá, 2015. p. 1-37. 